\title{
Patient handover in orthopaedics, improving safety using Information Technology
}

Tim Pearkes

Southmead Hospital, NHS, UK

\begin{abstract}
Good inpatient handover ensures patient safety and continuity of care. An adjunct to this is the patient list which is routinely managed by junior doctors. These lists are routinely created and managed within Microsoft Excel or Word. Following the merger of two orthopaedic departments into a single service in a new hospital, it was felt that a number of safety issues within the handover process needed to be addressed. This quality improvement project addressed these issues through the creation and implementation of a new patient database which spanned the department, allowing trouble free, safe, and comprehensive handover. Feedback demonstrated an improved user experience, greater reliability, continuity within the lists and a subsequent improvement in patient safety.
\end{abstract}

\section{Problem}

Quality and concise handover between clinicians is essential for patient safety [1]. At this time of increased risk during the patient's pathway the handover sheet is an important adjunct helping to reduce this risk [2]. The handover sheet within elective orthopaedic ward care takes on a variety of formats, with Microsoft (MS) Word or Excel being utilised frequently. These are susceptible to the erroneous deletion of data and on occasions the complete document. The change of team to which the patient is allocated within the speciality can also lead to data loss when being deleted from one list and added to another. Documents are rarely automated, requiring time to be spent ordering the patients by location and formatting the document. Following the amalgamation of two services into a large, new hospital it was recognised that a complete solution was required for the day to day management and handover of elective patients.

\section{Background}

The GMC's Good Medical Practice states that doctors should share all relevant information with colleagues involved in their patients care within and outside the team, including when they hand over care and go off duty [3]. Poor handover leaves on-call weekend doctors with busier shifts, a more stressful time prioritising patients and tasks whilst "fire-fighting" the new issues as they arise [4]. Clear patient handovers with concise and necessary information only lead to time-efficient, safer ward rounds. The handover sheet allows a snap shot to the patient's problems, the ongoing plan, outstanding jobs, and other relevant information. Keeping them up to date can be time consuming and this normally falls to the junior doctors to undertake. Their importance is evident within the current rules for working hours where most juniors rarely work a "normal" week, which leads to increased frequency of handovers [2]. They are also crucial when changing from the well staffed week-day rota to the weekend on-call team who have not met all of the patients. At this time the handover list and the information contained within is relied upon heavily [5].

As afore mentioned, IT is recognised as being an important part of patient handover, providing uniformity, continuity and an audit trail $[6,7]$.

\section{Baseline measurement}

Research was conducted within the orthopaedic junior doctors cadre to establish any concerns as to the current documents being used to conduct patient handover (MS Word). A questionnaire was distributed among the doctors (Pre-intervention questionnaire). Replies were completed by 6 doctors.

Options on the questionnaire - dependant on question type - see pre-intervention questionnaire for further details.

\section{ALWAYS / MOSTLY / RARELY / NEVER}

$0-2$ / 3-4 / 5-6 / 7-8 / 9-10

The questionnaire demonstrated the following results (Median):

1. Handover lists were readily accessible - MOSTLY

2. Handover lists were regularly updated by the $\mathrm{SHO}$ covering those patients - MOSTLY

3. Handover lists were automatically arranged into sensible layouts - NEVER

4. Patient data was lost when patients were transferred from one list to another - 3-4 TIMES

5. Patients were dropped off patient lists accidentally - 5-6 TIMES

6. These patients were dropped off these lists predominantly at the WEEKEND

7. Handover sheets allowed time efficiency at the weekend RARELY

Comments from freehand notes: 
Only 1 person on each team could access the list at once

Messy spreadsheet rarely contained ward locations

There was a lack of continuity as to the layout of handover sheets between the four teams

Patients who switched team due to changing consultant had been taken off a list and not added to another resulting in them not being reviewed/managed correctly

See supplementary file: ds4728.docx - "Pre-intervention questionnaire"

\section{Design}

It was deduced that the solution for this issue was within a bespoke computer interface. The author had reasonable experience with MS Access and its functions, and chose to create a system that could be implemented and serviced without the hospital IT department's assistance.

A table was created within MS Access, on which the data was to be stored, this was to be the "foundations" of the database with the information it held being interrogated/updated indirectly by users.

Queries that could sort and display relevant data according to criteria were created to subdivide by Consultant and therefore into the four teams that made up the department.

A form was created to allow the information from the table, selected by a query, to be accessed by the teams and edited as required. Specific functions were embedded within these forms, allowing new patients to be added and patients to be discharged (a two stage process preventing accidental deletion). Movement between team lists was enabled using similar function enabled buttons.

Reports were created allowing team lists to be generated from information within the relevant form at the click of a button, this could then be printed. The patients on the list were ordered by ward and then by surname alphabetically. A weekend report was also available which allowed patient lists to be printed by ward. This enabled better time efficiency during an already busy shift avoiding checking of multiple lists. Reports were automatically headed with the date and had a list of useful telephone numbers within the footnote.

Finally the robustness of the Database was ensured, with a "Save and Close" button with a number of background functions running. It saved the document followed by closing the document. Each time the Database was closed it would save a copy of the background Table as an Excel spreadsheet to an archive folder allowing teams to check back through lists at a later date.

\section{Strategy}

PDSA cycle 1
The Pre-implementation questionnaire was produced and handed out to members of the team. This allowed for an assessment of the current state of patients lists and their uses and downfalls, enabling a set of criteria on which to improve. These criteria also formed that basis upon which the new database was subsequently built.

PDSA cycle 2

- Following feedback from the questionnaire and initial demonstration/teaching session a number of ideas had been put forward:

- Multi-user functionality was poor and needed improving.

- A couple of program issues had been identified preventing the smooth running and failing its aim of being a robust system.

- A few spelling corrections were required!

These were all addressed, with multi-user functionality being addressed by the "splitting" of the database, programming issues (navigation buttons not working correctly) were resolved. Spelling errors were corrected.

PDSA cycle 3 - The database was again demonstrated to the junior doctors. On this occasion there were deemed to be no new issues. It was subsequently run in parallel with the old (MS Word) system for a two day period allowing for a safety net should any major errors arise. From this point onwards, no further formal updates to the program were required but instead as minor issues were found they were corrected on the live system.

PDSA cycle 4 - The questionnaire used in cycle 1 was again distributed. This revealed a general improvement across all aspects along with patient safety.

See supplementary file: ds4735.docx - "PDSA Cycles 1-4"

\section{Post-measurement}

Whilst information for the QI project is somewhat opinion based it has been formalised into a questionnaire in order to try and quantify/qualify these opinions. Indeed there is no obvious measure that can demonstrate an improvement in handover other than the lack of adverse incidents and near misses. When patients are found on the ward having been dropped from a list the doctors instinct is not to tell the author about the case but to ensure the patients safety and catch up on anything outstanding in the patient's care.

Following the implementation of the database the questionnaire was re-distributed with the results summarised as follows: The handover list was now always accessible and was no longed dependant on another doctor not having the document open simultaneously. The automated layout was sensible and continuous across all four teams. The incidence of patients and/or patient data being dropped from these lists had fallen dramatically with the discharge of a patient from the database now requiring a two stage process. Changing of team produced no loss in data as this was 
BMJ Quality Improvement Reports

done by changing the allocated consultant within the database. The "Print all patients" button allowed for all elective inpatients to be listed by ward and then surname order allowing for a logical and time efficient ward round.

See supplementary file: ds4741.pdf - "Database screenshots"

\section{Lessons and limitations}

When the system was taken "online" as the sole patient handover management system there were a number of issues that had to be resolved and this was done so whilst the author was in work. A problem arose a couple of weeks further down the line whilst the author was not in work. The result of an error within the spelling of a consultant's name had led to a patient being left off a list. This was one of the specific aims this project was seeking to resolve, thankfully the patient's team were aware of this and flagged it up to the author resulting in a further review of all spellings to ensure this didn't happen again.

This kind of project needs to be future proofed, allowing someone who is less IT literate to manage its upkeep once the author has moved on.

\section{Conclusion}

Reviewing the issues that led to the creation of this database:

- There was a lack of continuity as to the layout of handover sheets between the four teams

- Patients who switched team due to changing consultant had been taken off a list and not added to another resulting in them not being reviewed/managed correctly

- When carrying out the weekend ward round of all elective patients there was no easy way to print the patients by ward and this involved spending time studying lists to ensure no-one was missed

It was felt that the database had improved continuity for the junior doctors, with one departmental solution instead of a single list for each team. Patients were far less likely to be dropped from lists. Weekend elective ward rounds were more manageable with easier navigation of the lists and subsequent improvement in economy of time.

\section{References}

1. Acute Care Toolkit 1: Handover. London: Royal College of Physicians. May 2011

2. Safe handover: Guidance from the Working Time Directive working party. The Royal College of Surgeons of England. March 2007

3. Good Medical Practice, Domain 3:Communication, partnership and teamwork, continuity and coordination of care, para 44.a. March 2013

4. Ashton C. Improving weekend patient handover. BMJ Qual
Improv Report 2013;2:

doi:10.1136/bmjquality.u201303.w827

5. Curtis O, Fisher R. Improving medical SHO weekend handover at a tertiary referral centre. BMJ Qual Improv Report 2013:2: doi:10.1136/bmjquality.u697.w971

6. Till A, Sall H, Wilkinson J. Safe Handover: Safe Patients The electronic Handover system, BMJ Qual Improv Report 2014;2: doi:10.1136/bmjquality.u202926.w1359

7. Safe handover: safe patients. Guidance on clinical handover for clinicians and managers. London: British Medical Association. July 2004

\section{Declaration of interests}

nothing to declare 